\title{
River pollution models as an instrument for basin management
}

\author{
G. Genon \\ Politecnico di Torino, Italy
}

\begin{abstract}
River models are considered a useful instrument in order to assess the effects of point and non-point loads on receiving river waters. The problem of load allocation and definition of wastewater treatment limit can be conducted on the basis of use of predictive models, by evaluating real verification of effects.

The structure of models, the methodology for their validation and calibration are discussed, and the use of the obtained predictive tool is shown; applications are presented with reference to equilibrium between benefits from improved water quality, with lower externalities, and increased treatment cost. Also the problem of load reduction sharing between different subjects is presented, and attribution criteria are discussed.

Keywords: models, territorial program, wastewater, cost-benefits, externalities, treatment cost.
\end{abstract}

\section{Introduction}

In order to save the quality of water resources for future generations, and at the same time in order to allow the actual uptake for different uses, the European Directives (in particular Directive 2000/60/EC [1]) individuated quality standards for river waters, that can be respected in a scheduled time. The indications of these objectives have been translated in national laws, and also Italian law presents some examples of this application.

The Authorities in charge to program hydrologic basins destination have the burden to fix wastewater discharge limits for municipal treatment plants, to admit in water bodies industrial wastes with different final quality in consideration of realised treatment, to evaluate the effects of run-off phenomena on non-point immissions, to fix limits for agricultural use of 
potentially dangerous substances; from a more general point of view these indications must be done taking into account the necessity to respect prefixed criteria for acceptance, and to define this acceptance the scenario of allocation of loads must be drawn up.

In the river models both general parameters of organic pollution (BOD, suspended solids, nitrogen in different forms) and parameters of specific toxicity (heavy metals, solvents, micropollutants) are considered; by using the model approach it is possible to arrive to the load allocation, taking into account the correlation between applied loads and arising quality, on the basis of hydraulic river parameters and also kinetic parameters describing biological and chemical phenomena that naturally are occurring in water; the models therefore can be considered the fundamental support in order to establish acceptability scenarios. In the present work a methodological approach will be presented: basis, critical aspects and possible results of models are discussed, and as a conclusion the criteria utilised to apply this methodology will be treated, chiefly by taking into account the fundamental aspect of cost-benefits alternative.

\section{Individuation of load scenario}

The first subject to be dealt with for the application of the required methodology consists in the right definition of the introduced loads in receiving water bodies; it is very important to take into account that it is necessary to consider not only point but also non-point loads, and this fact is particularly significant with regard to some general pollution parameters (organic substances, nitrates, suspended solids).

As concerns point loads, it is in general possible to determine the value that must be attributed in connection with anthropic activities responsible to produce wastes introduced through wastewater flows in sewer systems (daily values of BOD for each inhabitant, as an example, or quantities of COD per ton of produced paper); these values can generally be derived from specialised references, or they can be obtained by means of emission factors; in general it is possible to conclude that an experimental study or determination are not required; only a verification of the local values in comparison with general ones can be in some cases required.

Afterwards it is necessary to apply to these estimated loads different values of removal efficiency, by considering the plant technological scheme that is in the particular case suitable to be used, and also taking into account cost considerations; about this, a specific discussion will be developed in a following point. On the basis of calculated original loads and removal efficiency hypotheses an emission scenario arises, and from this we can obtain a representation of the point waste fluxes in receiving waters. An example can be observed in [2].

The determination of non-point, diffused loads is absolutely more difficult, as illustrated from Becchi et al [3]; in fact it is quite impossible to determine these loads with an experimental procedure, and also a model estimation, that can be considered the only useful tool to arrive to these values, is rather complicated: it 
is possible to arrive to some estimations on the basis of definition of dynamics of hydrological exchange between connected aquifer and river body, taking into account the pollution concentrations that are present in both systems; as an alternative, with a more phenomenological approach, it is possible to pass through the evaluation of conveyed loads along the river, and subsequently to determine variations in this parameter; the estimation of the conveyed load in some consecutive river stations, by correcting in any case the obtained result in consideration of point loads, can lead to an indirect estimation of non-point loads; it is in any case necessary to recognise that the determinations are very complex, and chiefly that the data reliability seem to be in general fairly low.

Another important aspect must be considered in the definition of introduced loads: in fact the possibility to establish a relationship between point and nonpoint loads, with reference to technological schemes in the first case, to territorial technologies or practices that can be applied at the source in the second, is different. As concerns point loads having a derivation from households or industrial sewer system, the definition of a specific numerical value of improvement in technological load removal corresponds to the results of adoption of treatment apparatus that can be completely defined in order to obtain required results. For the diffused sources on the contrary it is quite difficult to establish a numerical relationship between modification of agricultural practices, or better management of urban areas where a run-off phenomenon can be expected, and exact value of point or non-point loads that from these sources will be immitted in receiving waters.

As a conclusion for non-point loads, in some cases it can be possible to arrive to an estimation of actual values of loads, and also to an evaluation of the influence of metereology on these loads, in account of different defluxion and elution phenomena, but it is very difficult, quite impossible to individuate the potential variation of loads as a consequence of different scenarios.

\section{Models for river phenomena}

Many physico-mathematical models have been defined and implemented in order to evaluate the correlation between point and non-point introduced loads and river quality at different stations and in different times; these models first of all consider the very high influence of the actual conditions of river stream, and afterwards take into account evolution phenomena of different complexity; in general, they have been completely developed as concerns numerical and resolution aspects and are reliable to allow a good predictive capacity.

The most precise models in particular, besides to advection, longitudinal dispersion and physical removal phenomena take into account the kinetic aspects arising from biological oxydation and algal growth operating in flowing waters, and also interactions with bottom sediments; the general structure of mathematical equations able to describe the whole mechanism is well known, but for each particular case it is necessary to define the exact value of kinetic parameters, and therefore a quantitative assessment can only be realised by 
using a model calibration on the basis of comparison between observed and calculated values; the value of the required kinetic parameters must be obtained by a backwards procedure.

Also with reference to persistent, not biodegradable pollutants and their fate some specific deterministic and stochastic models have been constructed and verified; the differences in these models in general arise from the fluidodynamic estimation of dilution phenomena, able to determine the final fate of immitted pollutants in river water [4].

A general consideration about models, both for biodegradable or for refractory parameters, is as follows: it is possible to use very simple models, that only take into account material balances between immitted loads and diluting effect of receiving water body, or to use as an alternative more sophisticated models, where the presence and influence of physical phenomena (settling, volatilisation), chemical phenomena (photochemical degradation, hydrolysis, precipitation) and biological phenomena (biodegradation, respiration, anaerobic digestion, nutrient assumption, algal growth) are considered, as it is illustrated in the exhausting review about models of Rauch et al. [5] the choice between simple or sophisticated models derives first of all from type of considered pollutant, but also from the possibility to have at disposal a reliable and verified data-base containing river experimental results; this data- base is a necessary tool in order to arrive to a calibration of the required model.

For the application of a predictive model to a specific river body, an experimental verification is necessary (of different complexity in account of different complexity of model structure), and at this regard it is very important to stress the very high importance of quality and quantity of data at disposal concerning the specific river stretch that is studied: first of all it is necessary to obtain values concerning river flow rate in different seasonal periods and at different significant stations of principal river and also affluents; at the same time, together with river flow rate determination, it is necessary to establish river quality and concentrations of desired components; the determination of concentrations must be directed to the definition of actual loads in different points of considered river stretches, and also to the determination of kinetics evolutions that are created along the river direction.

Very often it is possible to observe a situation of lack or low reliability of data at disposal, rather than difficulty in finding, choosing and solving river models; this seems to be the principal reason for the generally low knowledge of specific river model representations; for this reason it is quite difficult to use an useful predictive tool in order to introduce in it the load scenario estimations, or the application of this method can only represent a formal application, without real correspondence to real operating phenomena.

\section{Costs for limitation and environmental benefits}

By using a model that has been calibrated for the specific local river condition it is possible to arrive at predictive scenario considerations: in particular it is 
possible to make a comparison between different hypotheses of limitation of immitted loads, with deriving consequences in terms of quality of receiving body.

This comparison can lead to an immediate application, when it is possible to find and use quality standards for the receiving system, based on different water uses: in this case it will be possible to use this instrument to define the values of limitation of added loads able to realise a required result in terms of water quality.

In order to obtain an useful tool for load reduction policies, it is possible to use this predictive tool to find an equilibrium point between costs and benefits; in fact if cost data for different options of load reduction are at disposal, and also are at disposal the data concerning the benefits of lower externalities as a consequence of an estimated improvement of receiving body water quality, these two informations can be compared with an homogeneous numerical criterion; economic value of water resource and intervention cost are considered, in order to identify an equilibrium point.

As concerns the costs for limitation, their determination is rather simple and direct with regard to point load interventions; in fact from the usual experience in industrial engineering and sanitary environmental applications it is possible to extract a quite precise forecast about the cost for an identified intervention; the evaluation of the necessary costs for an indicated limitation to immission from non-point loads is more difficult to be obtained, in consideration of the difficult estimation of costs arising from modified management practices; chiefly it is important to indicate the high difficulty, as we discussed in the previous point, to connect different operating procedures with different values of introduced pollutant loads.

On the contrary some informations can be found about the environmental benefits arising from the obtained quality levels [6]; these informations, that concern avoided primary resource treatment costs or improved possibility to use water resource, can be found in technical literature; they are strictly referred in application to the situation where they have been definished, and are applied to local situations; therefore it is rather incorrect to extrapolate numerical values fro one situation to another.

In any case they can be used to have an idea about the order of magnitude of the benefits that it is possible to estimate to arrive at.

\section{Optimal values for the resolution}

As we have seen at the previous point, it is necessary to accept an increase in operating industrial costs in order to obtain an increase of the removal efficiency of pollutants at their source, and in comparison with this increase it is possible to observe on the basis of the determined immission scenario a decrease in external costs (or in other terms a benefits improvement) that arises from the quality improvement corresponding to a lower pollutant immission. 
The numerical solution of the optimisation procedure can be performed on the basis of aspects of strict formality, but it must be taken into account, as a real problem, the difficulty in some cases to find exact numerical values for the two cost definitions; in general, the most important aspect to be discussed is the meaning of the optimisation procedure, or in other terms the evaluations that must be taken into account in order to compare the two plots of cost; in fact the costs that the private owner must support to treat in a better way his discharge or the general costs that the public authority in charge of local policies for decrease in non point immission must deal with are real costs, that today must be supported; they must be compared with benefits of a surely higher difficulty to be evaluated, probably operating in different times, and chiefly directed to different subjects in comparison with the subject that afforded reduction load costs.

The question of the right position of the limit between the two opposite aspects, growing treatment cost, decreasing externality cost, is a specific choice field for the public authority, that holds the burden for this definition; the choice in any case can be more substantial and realistic on the basis of a technical proposition of completely studied and economically structured scenarios.

\section{Evaluation of the best scenario}

The application of a predicting model, realised with the above indicated criteria and limits, is able to lead to the identification of an exact value of load, that must be respected in order to obtain a fixed value for the concentration of some pollutants in receiving water body, as illustrated by Fair et al [7]; on the contrary, when the situation of contamination arises from many introduced loads, and as a consequence it is considered the need to respect quality limits in many stations, the problem becomes undetermined; in this case it is possible that infinite solutions of different combination of loads can all be defined as suitable to respect the required quality criteria; in these cases it is necessary to establish the best solution to be chosen.

Different criteria can be used in order to choose the solution that can be accepted as the best one, and in this case it is necessary, from the mathematical point of view, to introduce a further bond to the problem, in order to arrive to the best load allocation.

Mathematical solutions of different complexity $(8,9]$ have been proposed in order to arrive at the solution of this optimal identification, but the most important point in the present wok to be put into evidence is the possibility and meaning to use different criteria, and as a consequence the results in terms of different characteristics of allocated loads, as initially indicated by Joshi and Modak [8].

In order to have a clarification about this point, we can consider the hypothesis of two point sources immitting the same pollutant in a river; for simplicity, if we consider a pollutant of conservative type, by means of a simple mass balance the effects of different strategies in limitation of immitted load can 
be forecast as concerns river concentration; therefore if the volumetric flow rates of the two sources are known and different, different conclusion can be obtained as concerns load limitation and allocation.

In fact it is possible to arrive to the following scenarios.

- It is possible to evaluate the total load that can be introduced in the receiving body in order to obtain everywhere the required quality, and afterwards to share this total load in equal parts between the two pollution contributors; as a consequence of this equality of introduced load at the interior of two different flow rates it can be observed a different level of final concentration that is required to the two subjects; this criterion can be considered as regressive as concerns technological optimisation, as it requires a very low level of removal to the low flow rate immissions, and on the contrary an high level of removal, may be difficult to obtain with an economic compatibility, to the high flow rate discharges; but, from the point of view of the real impact, it is the most reliable criterion in order to distribute the responsibility of impact between different subjects, and from there to state effective burdens for pollution removal.

- It is possible to fix for both wastewater fluxes the same final concentration, by calculating this value on the basis of required quality; this criterion creates different effects of the two immissions on receiving body, in consideration of different volumetric load and different dilution effect; it is necessary to take into account in this case that for each river flow rate it is necessary to recalculate the concentration limit; this criterion can be considered the most neutral and fair against the polluters, and at the same time it can be considered as a very useful tool in order to sustain the maximum allowable level of technological improvement of all the plants.

- It is possible, if the cost for each plant in order to obtain a fixed level of removal of pollutants is known, and this cost as a first approximation can be considered as proportional to the flow rate and inversely proportional to required final concentration, to find the best solution as a solution of the minimum total cost, taking in any case in account the bond arising from required quality; this criterion, if the specific costs for the plants are the same, corresponds to the choice to adopt same final concentrations (as it should be easily demonstrated) but if the costs are different, the plant affected by an higher cost will find an optimum for an higher final concentration value; in this case the operative costs for the plants are not in the same measure depending on the flow rate, and it is therefore obtained an unfair solution.

The criterion of minimum total cost can be considered in general as an economic advantage, in any case by considering at the same time the need to respect the river quality bond and therefore by assuming a well defined externalities benefit; at the same time, in order to give to each polluter the same economic charge, it should be possible to arrive to some form of emission trading between the two subjects, in order to obtain also a so defined fair 
solution. This fact can correspond to a more difficult control, and in order to make it possible it is also required strict cooperation between pollution producers, but it can correspond to a real economic advantage for the different partners.

\section{Conclusions}

The methodological approach that has been presented in the present paper can be considered a starting point for the programming activity of the public territorial authorities, in order to define intervention policies useful to improve the quality of receiving water bodies, or to evaluate alternative hypotheses for delocalisation.

The operating scheme has is starting point in definition of loads and of their technological or operative possibility of limitation, and from there, by means of use of a verified and calibrated river model, allows the evaluation of the corresponding river quality aspects; it can be utilised in two directions, namely it can move from the original information in definition of the sources, in order to establish which is the environmental benefit that can be obtained from a defined limitation policy, on the contrary it can moves in the opposite direction, by evaluating which limitations must be taken into account in order to respect defined river quality limits.

The tool that has been proposed can be considered as absolutely rational and probably useful in many practical applications, but some aspects of difficult implementation must be solved, as it is indicated in the following points.

- Evaluation of non-point loads, with reference to the increased use of chemical fertilisers and to the consequences of atmospheric pollution and pollutant fall-out in urban areas; these phenomena produce, through the rain-out and aquifer immission, and river-underground transfer, effects of an extension probably important but at the moment rather difficult to be evaluated.

- Calibration and validation of many models that can be found in reference literature: for this operation it is necessary to have reliable experimental data about river quality, time evolution of riverine dynamic phenomena, hydrological aspects, and it is necessary to obtain a strict coordination with regards to obtaining data and general planning of field experimental activity.

- Definition of economic industrial charges to be sustained in order to realise the treatment systems of higher efficiency and to modify operating procedures; this argument is well known and completely developed from a technological point of view, but some considerations about acceptance of costs is strictly required.

- Definition of environmental benefits arising from an higher public water quality, in order to obtain better use possibilities and lower primary treatment costs; these aspects are more known as required best practices than as externality costs, and this last aspect must be more deeply studied. 
- Individuation of criteria useful to share the loads to be allocated; these criteria must take into account the aspects of fair attribution of charges, correct definition of real impact, possibility to have an advantage from compensating effects between different polluter subjects in order to obtain as a whole a benefit.

It is therefore possible to conclude that there is a rich knowledge on the subject, and from the other side that there is the absolute necessity for the public authority to use these instruments for river basin programming activity; these two moments must be conjugated trough technical definition, by taking into account that any solution must be constructed with reference to a specific local situation.

\section{References}

[1] Directive 2000/60/EC - EU Water Framework Directive

[2] T. Ramjeawon, J. Baguant - "Evaluation of Critical BOD Loadings from Mauritius Sugar Factories to Streams and Standard Setting"- Journal of Environmental management (1995) 45, 163 - 176

[3] Becchi I., Caporali E., Castelli F., Lubello C., Preti F., Settesoldi D. "Distributed geographic information for nonpoint source pollution modeling at high space - time resolution" - in 1995 Bouyoucos Conference, May 1-3, 1995, Riverside, California, pp.127 - 141

[4] A.M.J. Ragas, R.S.E.W. Leuven - "Modelling of water quality-based emission limits for industrial discharges in rivers" - Wat. Sci. Tech. Vol. 39, n. 4, pp. $185-192,1999$

[5] W. Rauch, M. Henze, L. Koncsos, P. Reichert, P. Shanahan, L. Somlyody, P. Vanrolleghem - "River water quality modelling: I. State of the art" Wat. Sci. Tech. Vol. 38, n. 11, pp. 237 -244, 1998

[6] Foundation for Water Research, U.K. - "Assessing the benefits of surface water quality improvements manual" - manual FR/CL0005, December 1996

[7] Fair G.M, Geyer J.C., Okun D.A. - "Wastewater Engineering" - New York, John Wiley and Sons, 1968

[8] V. Joshi, P. Modak - "Heuristic Algorithms for waste load allocation in a river basin" - in IAWPRC Conference "Water Pollution Research and Control", Brighton, July 1988, Proc. pp. 1057 - 1064

[9] R. Gu, M. Dong - "Water quality modelling in the watershed-based approach for waste load allocations" - Wat. Sci. Tech. Vo. 38, n. 10, pp. $165-172,1998$

[10] S. Karmakar, P.P. Mujumdar - "An inexact optimisation approach for river quality management" - Jnl. of Environm. Management 81, pp. 233 248 (2006) 